\title{
The Trivellini System and Technique
}

\author{
Roberto Trivellini, MD I Asuncion, Paraguay II Irtrivellini@yahoo.com.ar
}

Disclosure: Dr. Trivellini is the owner of Mamba Instruments, which is the manufacturer of the device described in this article.

The excision of follicular units using conventional FUE techniques has challenges and requires certain skills on the part of the physician. The multiphasic suction-assisted FUE device, described below, allows us to remove grafts with more tissue around the bulbs, less transection, and greater speed.

\section{BACKGROUND}

In conventional FUE, the physician needs to calculate and adjust his or her moves from area to area. The forces used to excise the follicular units need to be repeated many times for each patient. The punch must be aligned in the correct direction and at the correct exit angle of the scalp hair. This also depends on which position the physician adopts with respect to the patient. Next, the punch is advanced through the layers of the scalp. This could be challenging depending on such variables as the type of edge and geometry of the punch as well as the characteristics of the patient's skin and hair. Once the punch is through the scalp layers, the operator must decide when to stop the punch motion and when to retract.

Advancing the punch more than needed increases the transection rate, while not advancing it enough makes it impossible to liberate and remove the graft. It is very difficult to have control over these variables during FUE surgery. The Trivellini method was developed as a solution for reducing these variables. The system allows for repetition of the same sequence of action for every graft, thousands of times a day, and can work on any type of skin and hair. In addition, trauma to the follicles is minimized by directing the graft towards the center of the punch. Suction and vibration are two essential components of this system that work well with the potential energy that is generated by the follicles. In fact, the suction and the availability of the vibration in the system allows the operator to have maximum flexibility to consistently harvest high-quality grafts.

\section{CONSIDERATIONS}

If a scalp is divided scalp into 3 layers of $1.5 \mathrm{~mm}$ each, within the first two superficial layers, the densest ectodermal tissue with greater cell cohesion exists and decreases as you descend. Elastic fibers constitute $1 \%$ of the volume of the dermis and can be lengthened up to $99 \%$ or more before returning to their original lengths. ${ }^{1}$ The more superficial fibers are arranged perpendicular to the surface of the epithelium. In the deep layer, those elastic fibers become more disorganized. The bulb of follicle, in the third layer, is surrounded by adipose tissue. This layer is semi-liquid with a density of approximately $0.9 \mathrm{~g} / \mathrm{ml}$.

In classic FUE, when the surgeon introduces the punch into the skin, the punch undergoes permanent alterations in its trajectory. Once it crosses the glandular level, it enters the loose connective and adipose tissue. If the operator does not stop its advancement, the punch speed increases as it faces less resistance. When this happens, the trajectory of the punch becomes erratic, which increases the possibility of injury to the follicles. The Trivellini system provides a multiphasic approach that adjusts the punch mode and speed of motion as it enters different layers of scalp.

\section{METHOD}

\section{Suction}

Suction is a crucial element in this method. A pump that generates $650 \mathrm{mmHg}$ of vacuum is used. There is a certain relationship between the suction pressure (in $\mathrm{mm}$ of mercury indicated on the suction pump) and the suction flow at the tip of the needle. The exact pressure at the tip of the punch depends on the diameter of the tube system, punch, and device configuration. In a straight handpiece, a simple change in the diameter of the punch can double the amount of fluid aspirated with the same vacuum level.

\section{Device modes}

The Trivellini device provides a wide variety of modes. This versatility makes the device very flexible in dealing with different skin consistencies and hair characteristics. The device offers several modes such as rotation, oscillation, vibration, and oscillating asynchronous variation modes. It is possible to sequentially combine two or three modes and adjust the force and timing of each phase. The sequence of actions can be activated either by a slight touch of the pedal or automatically through the device's SmartReact ${ }^{\mathrm{TM}}$ system.

\section{SmartReact}

SmartReact is an intelligent computerized system that activates the motor when the tip of the punch is positioned over the follicular unit. The operator only needs to focus on following the exit angle of the follicles and placing the tip on the skin. The device does the rest automatically. (See video at the following link: https://www.youtube.com/watch?v$=$ Fl3R93XbrKY.)

\section{Time adjustment}

The duration of each mode can be adjusted much like the power. The operator can set up the system to function with several modes for a set power and duration. For example, the punch can start rotating with maximum power and after 200 milliseconds (ms) decrease the power by half for another $200 \mathrm{~ms}$. During this time, the punch will penetrate up to $2.5 \mathrm{~mm}$ and finish the scoring with less force.

The surgeon must decide among many possibilities to determine which is the best configuration for the type of skin encountered. The Trivellini system has a variety of punches, such as edge out and flared, in different sizes. Combining the versatility of the modes with the appropriate punch makes it very easy in almost every scenario to get high-quality grafts consistently. ${ }^{2}$ 


\section{RECOMMENDED PRACTICES \\ Surgeon position}

Our handpiece is not a modified dental instrument and was developed exclusively for hair transplantation. It is the only straight handpiece with a hollow shaft on the market and was created to be able to locate the handpiece between the point of view and the objective. This provides the surgeon with a three-dimensional view of the hair's exit angle, reducing the possibility of follicle transection (Figure 1).

FIGURE 1. Position of patient, surgeon, and handpiece for correct alignment and three-dimensional view of follicle's exit angle
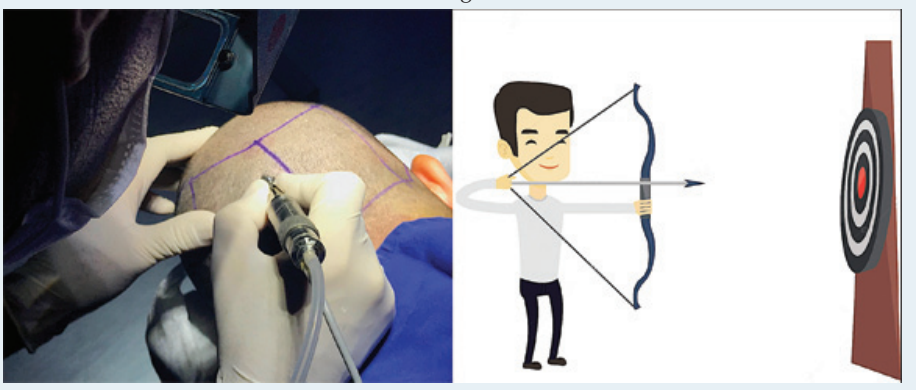

\section{Patient position}

The Trivellini system can be used with the patient in any position, however, to be able to place the surgeon behind the handpiece, we designed a bed the allows lateral and forward flexion of the head for better alignment of the handpiece with hair exit angle. The patient's arms hug the bed, so the operator can get close to the head (Figure 2). The operator is located to the side of the patient as shown in Figure 1.

FIGURE 2. Lateral and forward flexion of the head for better alignment of the handpiece with hair exit angle

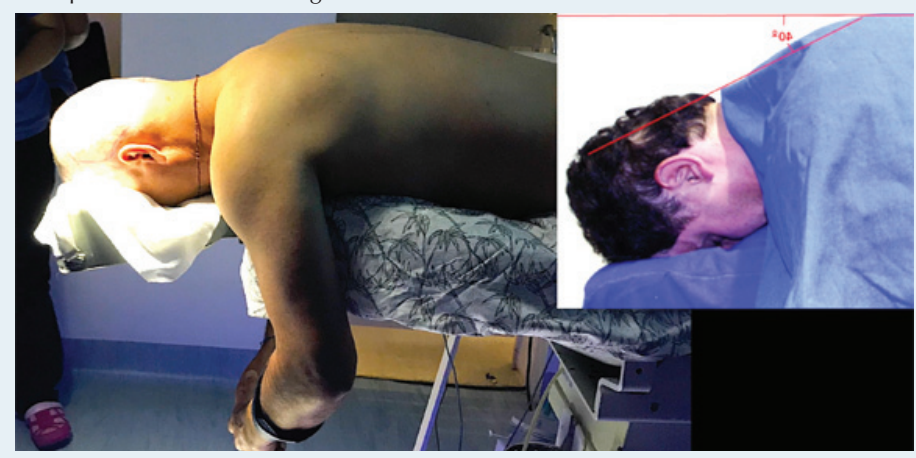

\section{INVOLVED DYNAMICS \\ Device configuration}

The device has many variables that need to be set by the surgeon for the optimum result. There are presets that work for most of the cases, but the surgeon may need to adjust the settings in more challenging cases. Following the skin consistency in different levels, one of the most common settings of the systems is rotation for $200 \mathrm{~ms}$ on high power and then switching to oscillation for $300 \mathrm{~ms}$. This generates a lot of vibration in the handpiece. (See video at the following link: https://www.youtube.com/watch?v=0RabXFW0-r0.)

This configuration allows the punch to penetrate the epidermis and the superficial dermis. These are the layers with greater resistance and rotating for $200 \mathrm{~ms}$ is the best movement to go through these tough layers. From this point, the oscillating movement is the most convenient to avoid twisting or damaging the graft.

\section{Dissection}

Once the punch has been centered over the follicular unit, the surgeon must hold the handpiece as close as possible to the tip, which provides for more control in positioning the punch. We recommend placing a finger at almost the same level as the punch as this allows a more precise targeting and positioning of the punch on the skin (Figure 3). The greater the distance between the grip of the handpiece and the scalp, the greater the distortion produced with the same movement.

FIGURE 3. The closer to the scalp the grasp on the handpiece, the
more accurate the approach to the follicular unit and faster movement more accurate the approach
to harvest the next graft

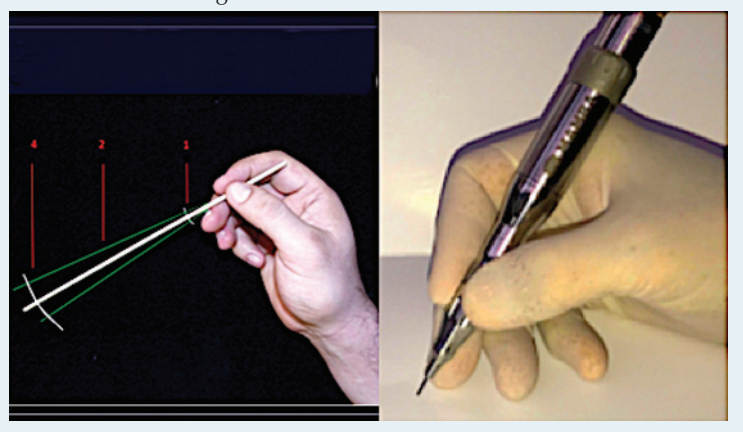

When the punch is positioned over the follicular unit, it should be held still. The suction makes a suction-cup effect on the skin, which ascends inside the punch to form a dome to the point where the pressure has been balanced. This immobilizes the punch on the surface and prevents the skin from moving when the punch begins to rotate. This is one reason that the operator doesn't need to use traction with the Trivellini system: the suction acts as the best stabilizer of skin and keeps it from moving away. The main reason, however, that we do not recommend applying traction is to facilitate the transmission of the vibration waves from the punch to the tissue, which allows the grafts to enter the interior lumen of the punch with minimal trauma. (See video at the following link: https://www.youtube.com/watch?v=hIR1hFO2Cm0.)

\section{Elasticity}

Elasticity is the ability of material to deform when receiving a force and then return to its original shape when the force ends. That is how the follicular units react when receiving the external force. After engaging with the skin surface, enough pressure must be applied to the punch to depress the surface of the skin about $2-2.5 \mathrm{~mm}$. This increase in axial force (gravity + pressure exerted by the hand) causes a transverse deformity perpendicular to the axis of the force. Traversing the epidermis to the deep dermis are hair shafts that have a different structure than the surrounding tissue. The shafts are formed by keratin (a protein with helical fibrous structure) and an amorphous matrix that keeps the microfibrils packaged. This gives the hair shafts a remarkable hardness and flexibility.

When follicles receive the axial load, they exhibit a phenomenon of elastic instability called "buckling." This means the follicle is loaded with potential energy, which is stored due to the deformation, until the punch cuts the upper layers of the skin and releases the follicles, which return to their original shape. (See video at the following link: https:// www.youtube.com/watch?v=ZVb4mZLcOgo.) 


\section{Dynamics of punch movements}

As the motor is activated by pressing the pedal or the

SmartReact, a sequence of events begins:

1. When the punch rotates, it cuts the epidermis and part of the superficial dermis during the first $200 \mathrm{~ms}$. This rotation engages the tip of the punch into the skin and cuts through the toughest layer of skin.

2. Next, the oscillation starts and is maintained for $300 \mathrm{~ms}$. Oscillation decreases the friction between the inner wall of the punch and the hair follicles.

3. The graft is then pushed into the punch by the potential energy of the hair. If the unit has two or more hairs, or if it is thick, the accumulated energy will be greater. Harris uses a hexagonal punch with 6 flat faces on the outer wall parallel to its axial plane that, when rotating, creates alternative waves throughout the surrounding tissue. The vibration reduces friction by continuous expansion and compression of the tissue that can help dissect the tissue from the external wall of the punch. On the other hand, as the hexagonal faces have a larger diameter than the cutting edge, the contact surface is reduced, which is very beneficial for the dull punch. ${ }^{3}$

4. The suction applied to the epidermis of the graft inside the punch generates an additional force that results in the graft entering the punch.

An important difference of this technique is that it does not require traction on the surface of the skin. ${ }^{4} \mathrm{On}$ the contrary, this suction-assisted system requires the follicular unit to move and vibrate so the natural forces can move the graft into the punch and dissect it from the surrounding tissue. When the surface of the scalp is tightened with traction to fix the follicles, the slightest contact of the sharp punch edge with a follicle will cut the follicle like a tense guitar string. We do not use deep tumescent infiltration since this decreases the density of the tissue, especially the adipose, and when using suction, the graft is aspirated. We do not want the graft to detach from the bed, therefore, we avoid deep tumescent infiltration, instead opting to infiltrate the superficial dermis, which tightens the surface and immobilizes the follicles inside the punch while leaving the deep part loose and avoiding the guitar string effect.

5. When 500 millisecond elapses, the movement of the punch stops. At this time, more than half of the graft is inside the handpiece, which has not moved, remaining in the same position. (See video at the following link: https://www.youtube.com/watch?v=297LKPis71w.)

6. Once the punch cuts the anchoring structures of the follicular unit, the graft is pulled upwards from the epidermis by the suction. As the lower part is fixed, the surrounding tissue is stretched so the punch can be advanced and cut through loose connective tissue and fat with the minimal force needed to cut the low-density hypodermis. With this dissection, more tissue around the stretched follicles is taken, creating chubbier grafts. With a punch of $0.90 \mathrm{~mm}$ diameter, we can obtain grafts with a base of $1.2 \mathrm{~mm}$ thickness (Figure 4). This also explains why splay is dealt with easily. When there is significant splay, the bulbs of the follicles of a unit are separated from each other in the deeper layers while the hair shafts are held together on the surface. It is not always necessary to advance the punch to this level, but when the anchor system is deeper, we have no choice. The operator can feel the resistance in this case. This feeling could be perceived through positioning the middle finger near the punch in contact with the skin and the fingers of the left hand on the scalp next to the extraction area. When the punch is advanced, a short advance movement is made while the fingers of the operator rest next to the punch as a pivot point. (See video at the following link: https:// www.youtube.com/watch? $v=3 \mathrm{HCuwINvcjl.)}$

7. When the punch is extracted from the scalp, the graft rises slightly, along with the punch, until the punch comes off when it loses its suction effect. The grafts stay loosely connected with a strand of loose connective tissue at their base and can easily and atraumatically be removed with forceps.

\section{CONCLUSION}

With my personal excision technique, I can obtain more tissue around and below the bulb, due to the traction exerted by the suction. The stretched follicular unit contracts at an angle perpendicular to the axial axis and allows the bulb to be extracted without depth control. The unit can easily be removed with just one forceps, and the total extraction time is greatly reduced due to the following:

- The punch has a thick wall and the geometry of its tip protects the follicles.

- The device executes programmable combined sequential movements that reduce the friction between the follicular unit and the inner wall of the punch.

- The suction increases the traction force in the epidermis of the follicular unit within the punch.

- The potential energy of the follicles is fully utilized.

For all this to work in an accurate and continuous way, it is necessary to keep the handpiece steady while the punch works, so that the follicular unit can go inside the punch. This is what ultimately prevents injuries to the follicles.

\section{References}

1. Wilkes, G.L., I.A. Brown, and R.H. Wildnauer. The biomechanical properties of skin. CRC Crit Rev Bioeng. 1973; 1(4):453-495.

2. Trivellini, R. An innovation in suction assisted FUE. Hair Transplant Forum Int'l. 2016; 26(2):58-59.

3. Harris, J. Blunt Hex Punch. Presented at the 2016 ISHRS 24th World Congress. Workshop 104: New Devices for FUE.

4. Mohebi, P. Dynamics of FUE. Hair Transplant Forum Int'I. 2017; 27(6):232-236. 